\title{
Shapes of geodesic nets
}

\author{
ALEXANDER NABUTOVSKY \\ REGINA ROTMAN
}

Let $M^{n}$ be a closed Riemannian manifold of dimension $n$. In this paper we will show that either the length of a shortest periodic geodesic on $M^{n}$ does not exceed $(n+1) d$, where $d$ is the diameter of $M^{n}$ or there exist infinitely many geometrically distinct stationary closed geodesic nets on this manifold. We will also show that either the length of a shortest periodic geodesic is, similarly, bounded in terms of the volume of a manifold $M^{n}$, or there exist infinitely many geometrically distinct stationary closed geodesic nets on $M^{n}$.

53C22, 53C23; 58E10, 58E35

\section{Introduction}

It has been a long-standing question in Riemannian geometry that originated with $\mathrm{H}$ Poincare whether every closed manifold has infinitely many periodic geodesics. Contributions to this question were made by W Klingenberg [8], and D Gromoll and W Meyer [4], who discovered that, on a closed Riemannian manifold, there exist infinitely many periodic geodesics if the space of parametrized curves on this manifold has an unbounded sequence of betti numbers. Subsequently M Vigue-Poirrier and D Sullivan showed in [14] showed that this condition is satisfied if and only if the rational cohomology algebra of $M$ requires at least two generators. In 1989 H-B Rademacher showed that, generically, there are indeed infinitely many periodic geodesics on any closed Riemannian manifold [11]. In 1992 it was shown by V Bangert [1] using the work of J Franks [3] that there exist infinitely many periodic geodesics on a manifold diffeomorphic to $S^{2}$. Later N Hingston found a lower bound for the number of geodesics of length $\leq x$ on a Riemannian 2-sphere [7].

On the other hand, for a manifold diffeomorphic to $S^{3}$, it is not known whether there always exist more than one periodic geodesic.

The question is complicated by the fact that for some nonsymmetric Finsler metrics on $S^{n}, \mathbb{C} P^{n}, \mathbb{H} P^{n}$ and $C a P^{2}$ only finitely many periodic geodesics do exist, as was shown by A Katok [8]; see also Ziller [15]. From this example one can conclude that, 
in general, the topology of a manifold does not by itself guarantee an infinite number of periodic geodesics.

Stationary geodesic nets can be viewed as a generalization of periodic geodesics. They are critical points of the length/energy functional on the space of graphs immersed in a manifold, similarly to periodic geodesics that are critical points of the above functionals on the space of closed curves. That is, if $\Gamma$ is a stationary geodesic net on $M^{n}$, then $\frac{d l_{X, \Gamma}}{d t}(0)=0$, where $X$ is any smooth vector field on $M^{n}, \Phi_{X}(t)$ is the corresponding 1-parameter family of diffeomorphisms of $M^{n}$, and $l_{X, \Gamma}(t)=\operatorname{length}\left(\Phi_{X}(t)(\Gamma)\right)$.

Every stationary geodesic net can be "made" into a stationary 1-cycle, if it is not already one, by doubling the multiplicity of each edge. Stationary 1-cycles can be considered as homological analogs of periodic geodesics.

Like periodic geodesics, two stationary geodesic nets are geometrically distinct if one is not a multiple of another.

Conjecture A On any closed Riemannian manifold there exist infinitely many geometrically distinct stationary geodesic nets.

Note that for a generic analytic Riemannian manifold the set of stationary geodesic nets is countable (for the same reasons that the set of closed geodesics is countable). Also, it seems that Morse-theoretic arguments do not help to conclude the existence of infinitely many geometrically distinct stationary geodesic nets in the situations, when they do not help to conclude the existence of infinitely many closed geodesics. For example, if $M^{n}$ is homeomorphic to $S^{3}$, then a well-known theorem by F Almgren implies that the space of 1 -cycles on $M^{n}$ is homotopy equivalent to $K(\mathbb{Z}, 2)=\mathbb{C} P^{\infty}$ and therefore does not have enough homology classes to conclude the existence of infinitely many stationary 1-cycles. Moreover, there are virtually no results establishing the existence of stationary geodesic nets that are not closed geodesics (or are formed by several intersecting closed geodesics); but see Hass and Morgan [6]. All this makes Conjecture A look as difficult for all practical purposes as the conjecture asserting the existence of infinitely many closed geodesics (although the latter conjecture is formally stronger). One of the objectives of the present paper is to indicate a possible approach to Conjecture A.

Another, seemingly different, open question is that of the connection between the length of a shortest periodic geodesic and other geometric parameters of the manifold. For example, one can ask whether the length of a shortest closed geodesic can be uniformly bounded in terms of the volume of a manifold (this question is due to M Gromov [5, 
page 135]) and whether the length of a shortest closed geodesic can be bounded in terms of the diameter of a manifold. Here is our conjecture:

Conjecture B On every closed Riemannian manifold there exists a periodic geodesic of length at most $c(n) d$, where $d$ is the diameter of a manifold.

It is worth noting that there is no known counter-example to show that the length of a shortest periodic geodesic is not, in fact, bounded by twice the diameter of a manifold.

Conjecture C [5] On every closed Riemannian manifold there exists a periodic geodesic of length at most $\tilde{c}(n) \operatorname{vol}\left(M^{n}\right)^{\frac{1}{n}}$, where $\operatorname{vol}\left(M^{n}\right)$ is the volume of a manifold $M^{n}$.

\subsection{Minimal geodesic nets}

In this paper we will prove two theorems: one of them stating that either Conjecture A or Conjecture B is satisfied for every closed Riemannian manifold $M^{n}$, and the second one stating that for a closed Riemannian manifold either Conjecture A or Conjecture $\mathrm{C}$ always holds. Moreover, our techniques are purely topological and apply also in the Finsler situation, even in situations when the set of distinct closed geodesics is known to be finite. Further more, if the assertion of Conjecture B (or Conjecture C) does not hold for a closed Riemannian manifold, then we demostrate the existence of infinitely many geometrically distinct geodesic nets on $M^{n}$, which are not periodic geodesics.

Minimal geodesic nets that result from the proofs of the theorems will be of a particular type. Namely, they will be minimal (stationary) geodesic cages and geodesic flowers formally defined below.

Definition $0.1 \quad[12 ; 13]$

(a) We define a minimal (or stationary) geodesic net $\Gamma$ to be a (finite) graph with edges enumerated by successive positive integer numbers $1,2,3, \ldots$ immersed into a Riemannian manifold $M^{n}$ that satisfies the following two conditions:

(1) each edge of $\Gamma$ is a geodesic segment;

(2) the sum of unit vectors at each vertex tangent to the edges and directed from this vertex equals to zero.

(b) If, in addition, all the vertices of $\Gamma$ have even degrees then $\Gamma$ is called a stationary $1-$ cycle. 


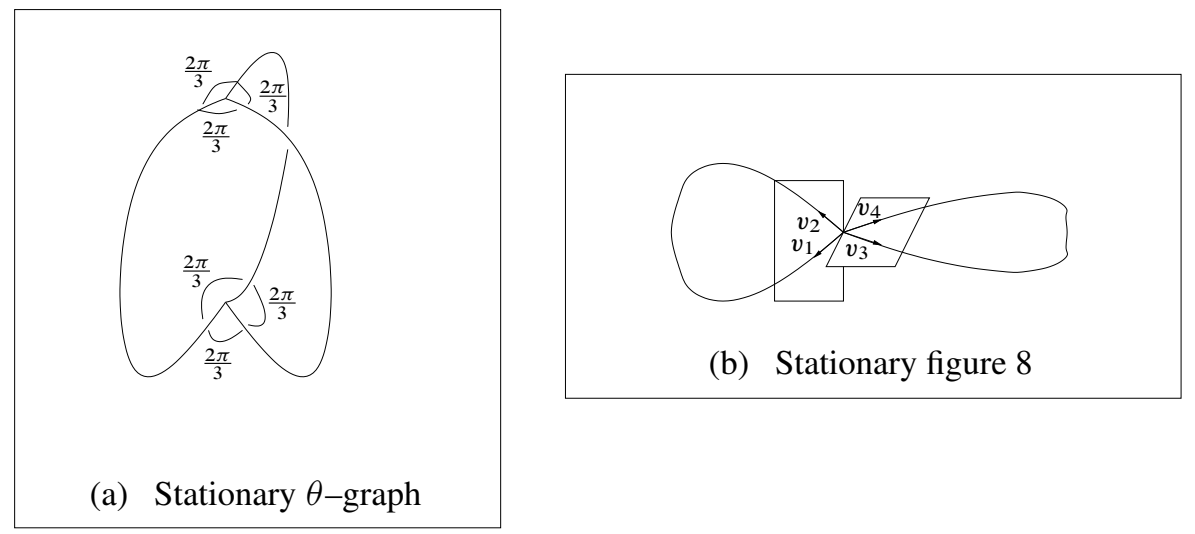

Figure 1: Geodesic nets

(c) If a minimal geodesic net $\Gamma$ has two distinct vertices joined by at most $m$ segments (counted with multiplicities) or if $\Gamma$ is a minimal geodesic flower, that is, a net that has one vertex and $m$ or less geodesic loops based at that point, it is called a minimal geodesic $m$-cage (or just a minimal geodesic cage) see Figure 1.

(d) A (not necessarily minimal or geodesic) immersion of a graph will be called a net. Also, nets that consist of a vertex together with at most $m$ (not necessarily geodesic) loops based at that point will be referred to simply as flowers and nets that are made of two vertices connected by at most $m$ (not necessarily geodesic) segments or nets that are $m$-flowers will be referred to as $m$-cages (or cages).

In this definition we allow a graph to have multiple edges between its vertices and to have loops. This object is sometimes referred to as a multigraph. Some of the distinct multiple edges can, in principle, be immersed by means of identical immersions. As the result, the images of edges of $\Gamma$ can have positive integer weights (greater than one). Therefore it is convenient to introduce notions of the carrier of a geodesic net which is defined as the set of curves in $M^{n}$ obtained as the images of edges of the multigraph, support of a geodesic net, which is the union of all geodesics from its carrier, and the multiplicity vector of a net, which is a vector with positive integer components indicating the multiplicities of geodesics in the carrier of the net. In this definition we completely disregard trivial edges (that is, edges mapped into constant curves). We do not include them into the carrier, and do not take them into account when we define the multiplicity vector. Two cages (or, more generally, nets) are geometrically distinct if either their carriers do not coincide or their multiplicity vectors are linearly independent (ie, are not (rational) multiples of each other). (See Remark 7 at the end of Section 2 for a discussion of this definition of geometrically distinct cages.) 


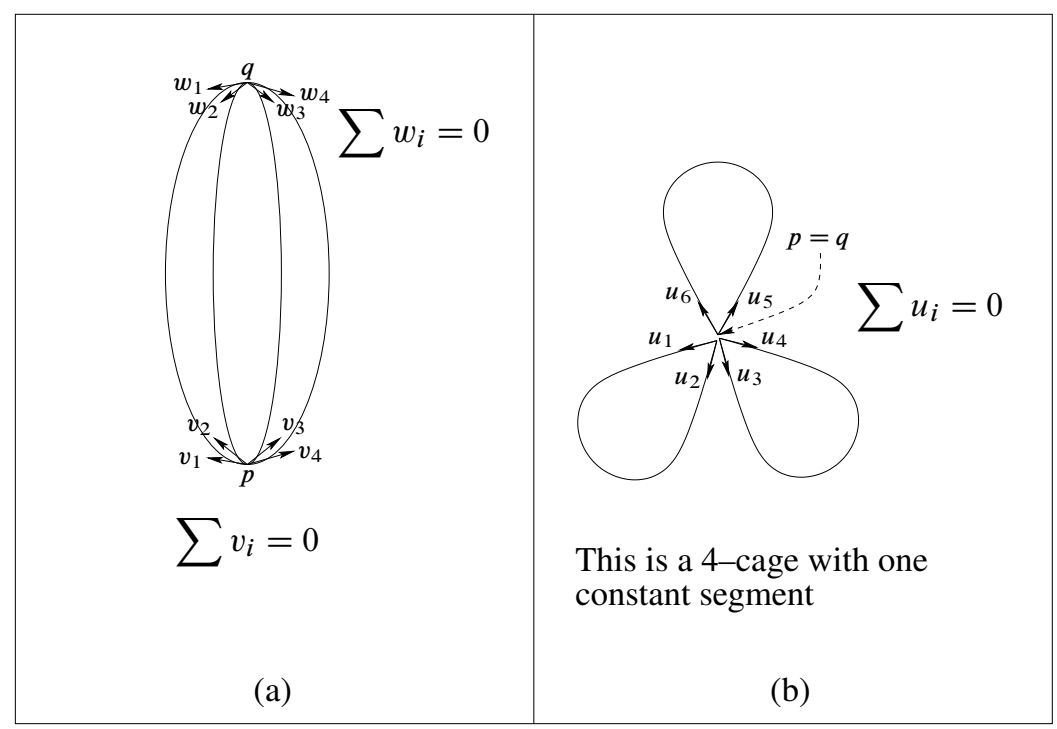

Figure 2: A non-degenerate stationary 4-cage and minimal geodesic 3-flower

Example 1 Obviously, a closed geodesic is a stationary 2-cage. It can also be considered as a geodesic flower with one petal. Geodesic loops are minimal geodesic cages if and only if they are periodic geodesics. A minimal $\theta$-graph will be an example of a stationary 3-cage, and so will be a stationary figure 8 . In the latter case two points $p$ and $q$ coincide and the length of the third segment equals zero. Some examples of stationary 4-cages can be found in Figure 2.

In [9] we proved analogs of Conjecture $\mathrm{B}$ and Conjecture $\mathrm{C}$ for integral 1-cycles. That is we obtained diameter (and volume) estimates for the smallest length of a stationary 1 -cycle.

In $[10 ; 12]$ we obtained similar estimates for $m$-cages. Finally in [13], we obtained similar estimates for geodesic flowers.

The main idea of the papers [10] and [12] can be summarized as follows. Let $f: S^{k} \longrightarrow$ $M^{n}$ be a map of the standard $k$-sphere into $M^{n}$. In the absense of (short) stationary $(k+1)$-cages one can construct a homotopy between $f$ and a sum of $k$-spheres in $M^{n}$, each of which is obtained by a "filling" of a $(k+1)$-cage in $M^{n}$. Then each of these $k$-spheres can be contracted to a point by contracting the corresponding $(k+1)$-cage to a point and filling the resulting cages by a $k$-sphere at every moment of the homotopy. (In the absence of short stationary cages these fillings can be performed continuously as a function of the parameter of the homotopy.) The $(k+1)$-cages are contracted 
using "the cage shortening process" similar to the Birkhoff length shortening process for closed curves, sometimes denoted as BCSP, (see [2] for the detailed description). Note, that if one applies a length shortening process to a (non-degenerate) $m$-cage, it is possible for it to degenerate into a flower. That is, the length of one of its edges can become zero, and the two vertices will then coincide.

The idea we used in [13] is that we can define a weighted length functional on the space of cages so that its gradient-like flow will "force" critical cages to degenerate into geodesic flowers. In other words, it can be arranged so that a (non-degenerate) stationary $m$-cage is not a critical point of the new functional.

\section{Definition 0.2 [13]}

(1) Let $\Gamma$ be a (not necessarily geodesic) net with edges $e_{1}, \ldots, e_{i}, \ldots, e_{k}$. Then $L(\Gamma)=\Sigma_{i=1}^{k} m_{i}$ length $\left(e_{i}\right)$, where $m_{i} \in \mathbb{Z}_{+}$and length $\left(e_{i}\right)$ is the length of the edge $e_{i}$ will be called a weighted length functional with weights $m_{1}, \ldots, m_{k}$. (Note that it corresponds to the regular length functional on the net, where each edge $e_{i}$ is taken with a multiplicity $m_{i}$.)

(2) A net $N$ is critical with respect to a weighted length functional $L$ with weights $m_{i}, i=1, \ldots, k$ if for any one-parametric smooth flow of diffeomorphisms $\Phi_{t}, t=0$ is a critical point of $\mu(t)=L\left(\Phi_{t}(N)\right)$. It is equivalent to all edges being geodesic segments combined with the following stationarity condition satisfied at every vertex of $N$ : the weighted sum of unit vectors tangent to edges of $N$ at that vertex and directed from it, equals to zero.

The new idea that we explore in this paper is that the weighted length functional can be applied repeatedly with different weights, not with the goal of obtaining flowers, but with a goal of obtaining distinct critical points.

Example 2 Let us consider the space of 3-cages and let $\Gamma$ be an element of this space. That is $\Gamma$ is a graph with two vertices $p$ and $q$ and three edges $e_{1}, e_{2}, e_{3}$. Define $L_{1}(\Gamma)=$ length $\left(e_{1}\right)+2$ length $\left(e_{2}\right)+3$ length $\left(e_{3}\right)$ and $L_{2}(\Gamma)=3$ length $\left(e_{1}\right)+$ 4 length $\left(e_{2}\right)+5$ length $\left(e_{3}\right)$. We claim that critical points of $L_{1}$ and $L_{2}$ are stationary cages that are geometrically distinct, unless it is a periodic geodesic.

To prove this claim let us examine their critical points. First, let us look at the possible critical points of $L_{1}$.

Note that a non-degenerate 3-cage can not be a critical point of $L_{1}$. Indeed, one of the conditions for it to be critical is that $v_{1}+2 v_{2}+3 v_{3}=0$, where $v_{1}, v_{2}, v_{3}$ are 
unit vectors tangent to $e_{1}, e_{2}, e_{3}$ respectively at $p$. Obviously, this condition can be satisfied if and only if the cage is a periodic geodesic. Therefore, if $\Gamma$ is critical then either one of the $e_{i}$ 's degenerates into a point and the cage degenerates either into a "figure 8" or a periodic geodesic. In the case of a "figure 8", the two loops can have the following multiplicities: (a) 1 and 2; (b) 1 and 3; (c) 2 and 3.

Next, let us look at the critical points of $L_{2}$. Its critical points can be

(1) a cage that consists of two vertices and three edges with multiplicities $3,4,5$, like in the original cage;

(2) a "figure 8" with one of the following multiplicities (a) 3 and 4; (b) 3 and 5; (c) 4 and 5;

(3) a periodic geodesic.

Even in the case of "figure 8", the stationary cages will be different, because the pair of multiplicities are not multiples of each other. Thus, if the appearance of periodic geodesics can be excluded, we will obtain different geodesic nets.

We then combine this idea with the techniques of [12] that will be explained in the next section.

Note that although the critical points of the weighted length functionals are not necessarily critical points of the length functional, one can make them into such by taking some of the edges with appropriate integer weights. Observe that if a stationary cage $C g$ that consists of $k$ distinct edges $e_{1}, \ldots, e_{k}$ is a critical point for the weighted length functional with weights $m_{1}, \ldots, m_{k}$, then a stationary cage $\tilde{C g}$ that consists of the geodesic edges $e_{i}$ taken with multiplicities $m_{i}, i=1, \ldots, k$ will be a stationary cage, that is a critical point for the regular length functional. This observation will be used throughout the paper.

A similar construction of a new "weighted" net from a given net and a given weighted length functional will be used many times below in this paper. Note that this construction is somewhat ambiguous, because all edges of nets must be numbered by consecutive integer numbers. So, when we replace an edge by, say, three geometrically identical edges, then according to our defintion we need also to indicate what are the indices of these edges in a numbering of all edges of the resulting graph. Yet we need to know these indices only to be able to calculate the weighted length functionals on the net. Since below we are not going to evaluate weighted length functionals on geodesic nets obtained by this construction, this ambiguity will not create any problems. To make everything rigorous, we could define also unordered geodesic nets, where edges of the underlying graph are not numerated. Then for every weighted length 
functional and every (ordered) geodesic net we can construct an (unordered) geodesic net by multiplying all edges of the given geodesic net by the weights prescribed by the weighted length functional. The weighted lengths functionals are not defined for such nets but the usual length functional is, of course, defined. But we do not want to make our system of definitions too cumbersome. Therefore we will be neglecting the distinction between ordered and unordered geodesic nets.

\subsection{Main results}

In the next section we will prove the following two theorems.

Theorem 0.3 Let $M^{n}$ be a closed Riemannian manifold of dimension $n$ and of diameter $d$. Let $q=\min _{i}\left\{\pi_{i}\left(M^{n}\right) \neq\{0\}\right\}$. Then either the length $l$ of a shortest closed geodesic is $\leq(q+1) d$ or there exist infinitely many stationary geodesic cages on $M^{n}$. Moreover, in the last case for every positive $\delta$ there exist infinitely many stationary geodesic cages with carriers that consist of at most $q+1$ geodesic segments of total length $\leq(q+1) d+\delta$.

Remark 1 It will be clear from our proof of Theorem 0.3 that there exists an increasing function $C(k, n, x)$, which can be explicitely calculated with the following property: If $l>(n+1) d$, then for every $k$ there exist at least $k$ distinct stationary geodesic cages of length $\leq C\left(k, n, \frac{d}{l-(n+1) d}\right) d$. (Of course, here we count the lengths of geodesics in the carrier with multiplicities coming from the multiplicity vector. So, we are basically saying that we are able to majorize the norm of the multiplicity vectors of the stationary geodesic cages, and not only the total length of their carriers.) As a corollary, if $l$ is greater than or equal to, say, $(n+2) d$, then there exists $\bar{C}(n, k)$ such for every $k$ there exist $k$ geometrically distinct stationary geodesic cages of length $\leq \bar{C}(n, k) d$. Our proof will produce infinitely many stationary geodesic cages that not only satisfy this length bound but also have carriers that consist of at most $q+1$ geodesic segments.

Remark 2 There are no known counterexamples to the conjecture that the length of a shortest closed geodesic is always $\leq(q+1) d$ (and even $\leq 2 d$ ). Morover, this conjecture can turn out to be true even in the Finsler case.

Theorem 0.4 Let $M^{n}$ be a closed Riemannian manifold of dimension $n$ and of volume $\operatorname{vol}\left(M^{n}\right)$. Then either there exists a periodic geodesic of length $\leq \widetilde{c}(n) \operatorname{vol}\left(M^{n}\right)^{\frac{1}{n}}$, or there exist infinitely many geometrically distinct minimal geodesic nets. If the length of the shortest periodic geodesic is greater than $\widetilde{c}(n) \operatorname{vol}\left(M^{n}\right)^{\frac{1}{n}}$, then for every $k$ there exists at least $k$ geometrically distinct minimal geodesic nets of length $\leq$ $\widetilde{C}(n, k) \operatorname{vol}\left(M^{n}\right)^{\frac{1}{n}}$. Here $\widetilde{c}(n)$ and $\widetilde{C}(n, k)$ are constants that can be explicitly written down. 
Remark 3 It can be arranged using the methods introduced in [13] that nets that appear in Theorems 0.3 and 0.4 would be stationary geodesic flowers. However, to achieve that, the bounds on the length of a shortest periodic geodesic should be made significantly worse (although, they still be of the form $c(n) d$ and $\left.\widetilde{c}(n) \operatorname{vol}\left(M^{n}\right)^{\frac{1}{n}}\right)$. See Remark 6 in Section 2 for a specific theorem in this direction.

In this section we will prove Theorem 0.3 in the case when $q=2$. Note that when $q=1$, the length of a shortest closed geodesic is bounded above by $2 d$, thus Theorem 0.3 is trivially satisfied.

Proof of Theorem 0.3 for $\mathbf{q}=\mathbf{2}$ Let $f: S^{2} \longrightarrow M^{n}$ be a non-contractible map from a sphere endowed with a fine triangulation (the diameter of each simplex $\leq \delta$ ) into $M^{n}$. The proof will be done by contradiction. That is, assuming there is no periodic geodesic of length $\leq 3 d$ and only finitely many geometrically distinct cages, we will extend the map $f$ to $D^{3}$ triangulated as a cone over the triangulation on $S^{2}$, thus reaching a contradiction.

The extension will be done as follows: it is trivial to extend $f$ to the $0-, 1-$, and 2-skeleta of $D^{3}$. We map the center of the disc, $\widetilde{p}$ to an arbitrary point $p \in M^{n}$, edges of the form $\left[\tilde{p}, \widetilde{v}_{i}\right]$ to minimal geodesic segments denoted as $\left[p, v_{i}\right]$ connecting the point $p$ with the vertex in the induced triangulation $v_{i}=f\left(\widetilde{v}_{i}\right)$ and, finally, we map the 2-simplices of the form $\widetilde{\sigma}_{i}^{2}=\left[\tilde{p}, \widetilde{v}_{i_{1}}, \widetilde{v}_{i_{2}}\right]$ to the surface generated by a length-decreasing homotopy connecting $f\left(\partial \widetilde{\sigma}_{i}^{2}\right)$ and a point. Here we are using the assumption that the length of a shortest periodic geodesic is bigger than $3 d$.

\section{Extending to the 3-skeleton (see Figure 3)}

To extend to the 3 -skeleton we will use a trick used in $[10 ; 12 ; 13]$, except we will use it infinitely many times. The trick is the following. Let $\widetilde{\sigma}_{i}^{3}=\left[\tilde{p}, \widetilde{v}_{i_{1}}, \widetilde{v}_{i_{2}}, \widetilde{v}_{i_{3}}\right]$ be an arbitrary 3-simplex. Its boundary consists of four 2-simplices glued in an obvious way. One of the simplices $\left[\widetilde{v}_{i_{1}}, \widetilde{v}_{i_{2}}, \widetilde{v}_{i_{3}}\right]$ and consequently $\left[v_{i_{1}}, v_{i_{2}}, v_{i_{3}}\right]$ is so small that it can be treated as a point $q$. This assertion can be made more rigorous (see Remark 4). Let us consider the image of 1 -skeleton of $\tilde{\sigma}_{i}^{3}$. Assuming $\left[v_{i_{1}}, v_{i_{2}}, v_{i_{3}}\right]$ is a point, the image of the 1-skeleton consists of three edges that we will denote as $e_{1}, e_{2}, e_{3}$ respectively, connecting $p$ and $q$. This is a 3-cage. This 3-cage corresponds, of course, to the 2 -sphere $f\left(\partial \widetilde{\sigma}_{i}^{3}\right)$ constructed from it by considering 3 of its digons and contracting each pair to a point by BCSP, (see Figure 3 (a)). The idea running through papers $[10 ; 12 ; 13]$ is that if we can contract this cage to a point, then we can contract $f\left(\partial \widetilde{\sigma}_{i}^{3}\right)$ to a point as well. 


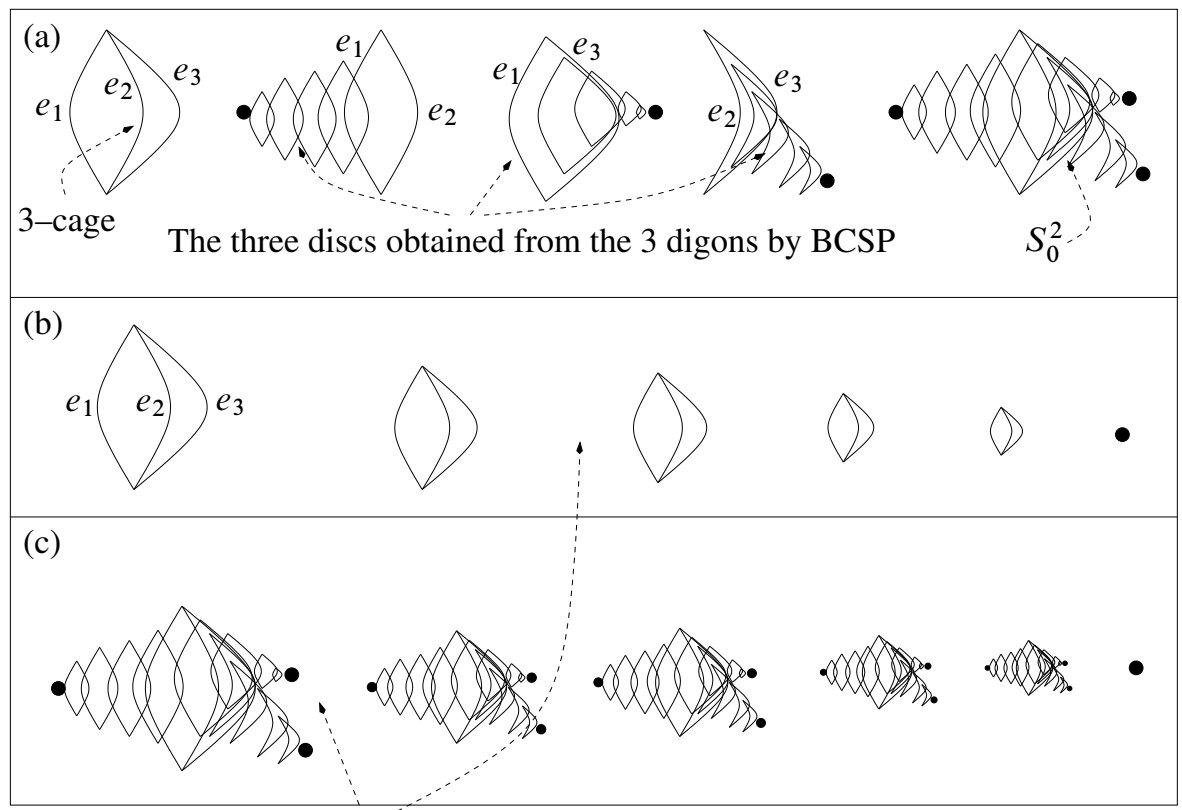

1-parameter family of 3-cages leads to 1-parameter family of 2-spheres

Figure 3: Extending to 3-skeleton

The new idea is that there are infinitely many ways we can try to contract this cage to a point. If one of them works then we have suceeded in extending the map to any 3 -simplex in the triangulation of $D^{3}$. If none of them works, then for each attempt we get a new obstruction, which happens to be a stationary 3-cage. As with geodesics, we should be careful that we, indeed, get geometrically distinct nets and not a multiple of the same net.

First, let us explain why it suffices to contract the 3-cage to a point. As we would like to extend our map to the 3-simplex, we want to construct a 3-disc that has $f\left(\partial \widetilde{\sigma}_{i}^{3}\right)$ as its boundary. This disc can be constructed as a 1-parameter family of spheres $S_{\tau}^{2}, 0 \leq \tau \leq 1$ that starts with the original sphere $f\left(\partial \widetilde{\sigma}_{i}^{3}\right)$ and ends with a point (see Figure 3 (c)). This one parameter family of spheres is constructed as follows: Let $N_{\tau}, 0 \leq \tau \leq 1$ be a 3-cage during some fixed deformation of the initial 3-cage to a point (see Figure $3(\mathrm{~b})$ ). We can consider three digons formed by $\left(e_{1}\right)_{\tau},\left(e_{2}\right)_{\tau}$, $\left(e_{2}\right)_{\tau},\left(e_{3}\right)_{\tau}$ and $\left(e_{3}\right)_{\tau},\left(e_{1}\right)_{\tau}$. Note that, it is possible that one or two of the segments have length zero. Each of these digons varies continuously with $\tau$. Moreover, each of these digons can be contracted to a point without the length increase, assuming there are no short geodesics, thus generating 2-discs. It is essential that those discs depend 
continuously on the initial digon. Next, at each time $\tau$ we glue the boundaries of the three discs that correspond to the three digons as in the boundary of the 3-simplex, obtaining $S_{\tau}^{2}$. Th fourth simplex in the boundary of the 3-simplex is shrunk to a point. Note also, that if we succeed at contracting the net to a point, then $S_{1}^{2}$ is also a point and, thus, we have obtained a 3-disc.

Next, we would like to describe the many ways to contract a cage. They correspond to many ways of assigning the multiplicity coefficients to the edges of the 3-cage that result in geometrically distinct stationary geodesic nets. To every weighted length functional $L$ we can assign a process that decreases the value of $L$ until it reaches a critical point. This process is defined as follows: For a given cage consider a cage made of the same geodesic segments, but with weights determined by the length funtional. Apply the "usual" length decreasing process (described in $[9 ; 10 ; 12 ; 13])$ to this new cage. Then we "forget" about the assigned weights at every moment of the constructed homotopy. The resulting cages will form a homotopy of our initial case which can end only at a critical point of the weighted length functional.

We, in fact, suggest a specific way of choosing the coefficients of the weighted length functionals that we will be using to contract 3-cages. Namely, we choose weights as $m, m+1, m+2, m \in\{1,3,5, \ldots\}$ for different values of $m$. We now would like to note that the infinite number of ways of assigning coefficients does, indeed, lead to infinite number of geometrically distinct critical poins and not just multiples of finitely many geometrically distinct critical points.

First, note that during the deformation of the net one of the following three things can happen:

(a) An edge can disappear Then the possible critical point will be a geodesic flower. In fact, it will be a figure 8 , of one of the following three types, depending on which edge disappeared: (1) one petal has multiplicity $m$ and the second petal has a multiplicity $(m+1) ;(2)$ one petal has multiplicity $m$ and the second one has a multiplicity $(m+2)$; (3) one petal has multiplicity $(m+1)$ and the second petal has multiplicity $(m+2)$. The question is for which different positive integers $m$ and $k$ the corresponding weighted length functionals result in geometrically the same nets. Assume $m>k$. Then getting the same net would mean that the following two equations have to be simultaneoulsy satisfied:

$m+x_{1}=\alpha\left(k+y_{1}\right)$ and $m+x_{2}=\alpha\left(k+y_{2}\right)$, where $\alpha$ is a positive integer, $x_{1}, x_{2}, y_{1}, y_{2} \in\{0,1,2\}$ and $x_{1} \neq x_{2}$, whereas $y_{1} \neq y_{2}$.

Analysing this equations we come to the conclusion that the following are the only non-trivial ways in which they can be satisfied by positive integers: 
(1) $\alpha=1, m=k+1$. This possibility can be excluded, once we assume that $m, k$ are odd integers.

(2) $\alpha=2, m=2 k$ or $\alpha=2, m=2 k+2$. This possibility is also excluded when $m$ is odd.

Thus, in the case of disappearence of an edge, our method does, indeed produce distinct cages.

Note, it is possible that after one edges disappears, the remaining two petals merge, but the only stationary geodesic net of this type would be a short periodic geodesic.

(b) Two edges disappear In that case the resulting stationary cage can only be a periodic geodesic. Its length is at most $\frac{m d+(m+1) d+(m+2) d}{m}=3 d+\frac{3 d}{m}$. As $m$ becomes large, this bound approaches $3 d$. This calculation has the following implication: Assume that the weighted cage shortening process gets stuck at a critical point that is a non-trivial periodic geodesic for infinitely many of the considered weighted length functionals. Then the infimum of lengths of these periodic geodesics does not exceed $3 d$.

(c) None of the edges disappear In that case one can easily see that the stationary points must be different for different integers.

\section{The scheme of the proofs of Theorems 0.3 and 0.4}

The rough scheme of the proof of Theorem 0.3 goes as follows. We begin with a non-contractible map of a sphere $S^{q} \longrightarrow M^{n}$ of a smallest possible dimension to a manifold $M^{n}$. Assuming the conclusion of Theorem 0.3 is not satisfied we will extend the map to a disc, thus reaching a contradiction. The extension process has a structure of $\mathbb{R}$-tree. Let us describe it in more detail (see Figure 4).

Step 1 First, let us note that it is trivial to extend the map to $0-, 1-$ and 2-skeleta of $D^{q+1}$. It will be done exactly as in the case of $q=2$ with which we dealt in the previous section. The only assumption that we will need to use is that there are no "short" periodic geodesics on $M^{n}$.

Step 2 In the previous section we also dealt with extending to the 3-skeleton. Recall that we found infinitely many ways of extending to the 3-skeleton. They correspond to infinitely many weighted length functionals, $L^{3}\left(m_{j}^{3}\right)$, on the space of 3-cages, where $\left\{m_{j}^{3}\right\}$ is a sequence of natural numbers. Note that for every $j L^{3}\left(m_{j}^{3}\right)$ is defined by 


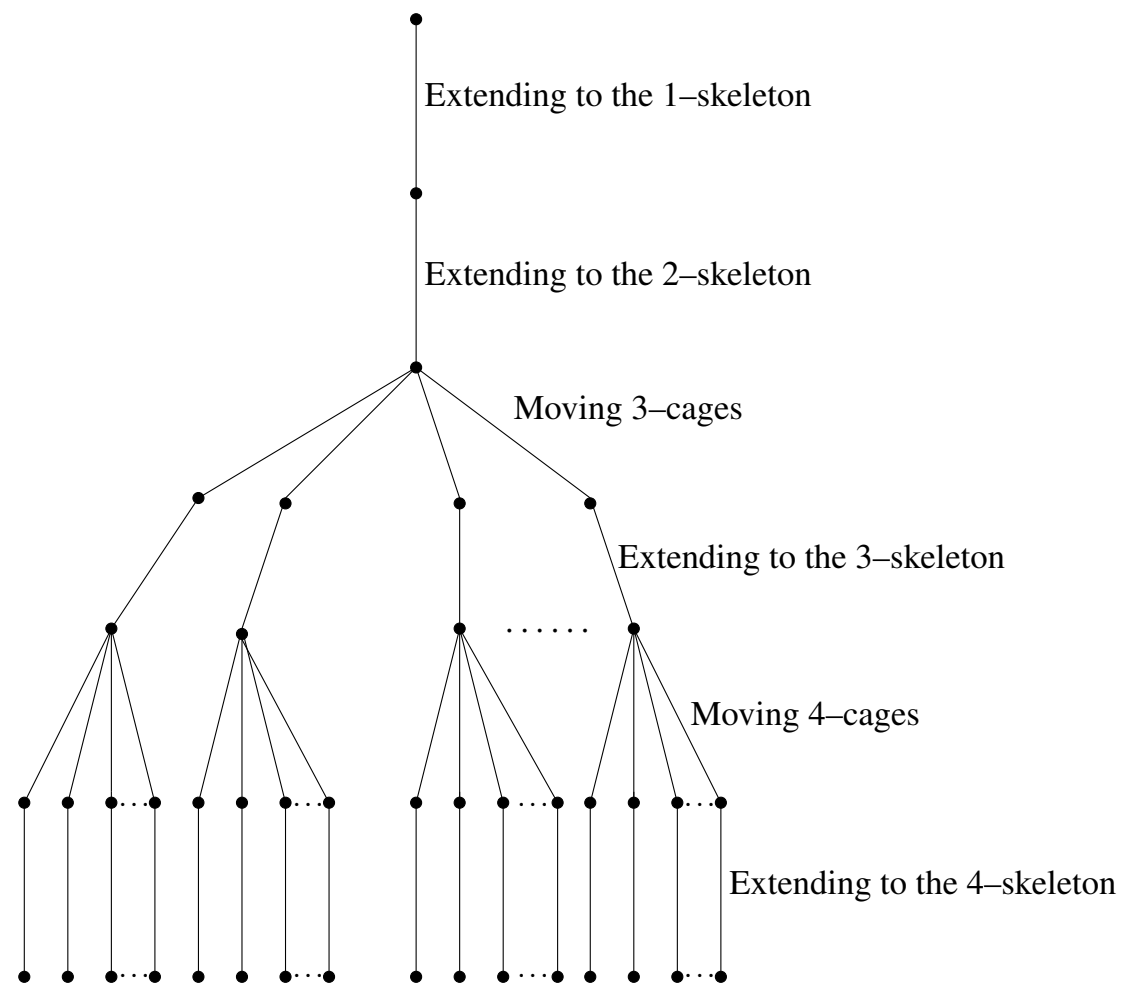

Figure 4: The scheme of the proof

three weights. We prefer to write these weights in the form $m_{j}^{3}+c_{i}, i=1,2,3$. We have demonstrated above that we can take $m_{j}^{3}=2 j+1$ and $c_{i}=i-1$.

Observe that either the contraction of three cages corresponding to $L^{3}\left(m_{j}^{3}\right)$, indeed, leads to extension to the 3 -skeleton, or it stops at a critical point of $L^{3}\left(m_{j}^{3}\right)$. We can assume that there are only finitely many critical points of functionals $L^{3}\left(m_{j}^{3}\right)$ that are not periodic geodesics, or we are done. This means that for most choices of $m_{j}^{3}$ the corresponding weighted length functional does not have critical points corresponding to cages that are not periodic geodesics. We are going to consider only these weighted length functionals. Further, if our extension attempt fails, it must fail at a periodic geodesic of length $\leq 3 d+3 d / m+\varepsilon$. If the extension attempts fail for infinitely many weighted length functionals, we obtain a periodic geodesic of length $\leq 3 d$. Therefore, choosing a cofinite subset of the considered set of weighted length functionals we can assume that all our attempts to contract each of the considered 3-cages to a point using one of the considered weighted length functional are successful. 
Let us represent every such weighted length functional (regarded as a way to extend the considered map to the 3 -skeleton) by a branch of the tree. There are infinitely many of such branches.

Step 3 Let us consider a particular extension to the 3-skeleton, let's say, $L^{3}\left(m_{*}^{3}\right)$. We will now extend the previous map to the 4-skeleton. Likewise, there will be infinitely many attempts, corresponding to different ways of contracting 4-cages in accordance with different weighted length functionals $L^{4}\left(m_{j}^{4}\right)$, where $\left\{m_{j}^{4}\right\}$ is a sequence of natural numbers. As before, every weighted length functional $L$ determines the following procedure of contracting 4-cages: Multiply all segments of a considered 4-cage by weights of $L$. Apply the cage shortening process to the resulting weighted 4-cage. Then "forget" about the weights that came from $L$ obtaining a homotopy of "unweighted" cages.

The weights of $L^{4}\left(m_{j}^{4}\right)$ will be of the form $m_{j}^{4}+c_{1}, m_{j}^{4}+c_{2}, m_{j}^{4}+c_{3}, m_{j}^{4}+c_{4}$. (See Section 2 for details how exactly we choose values of $m_{j}^{4}$ and $c_{i}$.) Those attempts will be successful, unless $L^{4}\left(m_{j}^{4}\right)$ has a critical point. We are yet to show that different weighted length functionals correspond to different critical points unless these critical points are (possibly trivial) closed geodesics. We will also show that if the extension process for infinitely many of these weighted length functionals get stuck at a nontrivial periodic geodesics, then the infimum of lengths of these periodic geodesics cannot exceed $(4+1) d$. These two facts together imply that either the assertion of the theorem is true, or infinitely many of these contraction attempts will be successful for all considered 4-cages. Each successful attempt to contract a 4-cage corresponds to a 4-disc "filling" this cage as follows. Consider a 1-parameter family of 4-cages $C g_{\tau}^{4}, \tau \in[0,1]$ that starts with our cage and ends with a point. At time $\tau=0$ it is a 1 -skeleton of a 3-sphere $S_{0}^{3}$. We can construct a 1-parameter family of 3-spheres that begins with $S_{0}^{3}$ and ends with a point. This family will generate a disc. Now each sphere $S_{\tau}^{3}$ is constructed from four 3-discs by gluing them as in the boundary of the four-simplex, but keeping in mind that the fifth disc is simply a point. 3-discs are constructed exactly as in Step 2 (using $L^{3}\left(m_{*}^{3}\right)$ ). That is, we consider four 3-cages obtained from $C g_{\tau}^{4}$, by forgetting one of the edges, to each of the triplets we apply the weighted length shorteing process associated with $L^{3}\left(m_{3}^{*}\right)$, etc. Step 2 is, thus, repeated at each $\tau$ giving the process the structure of an $\mathbb{R}$-tree.

We can proceed as above until we extend to the $(q+1)$-skeleton of $D^{q+1}$, reaching a contradiction.

Here are some principles on which the proof is based: 
Principle 1 Consider a non-contractible map $f: S^{q} \longrightarrow M^{n}$. The infinitely many distinct geodesic nets correspond to infinitely many attempts of extending this map to $D^{q+1}$. Of course, all of these attempts should fail. These geodesic nets are obstructions to extensions. They are the same only if they all happen to be the same "short" periodic geodesic. The last assertion is not automatic. The weights should be chosen very carefully, as it is described below, to make it true.

Principle 2 The infinitely many extension attempts correspond to infinitely many different weighted length functionals, which in its turn correspond to infinitely many ways of contracting $k$-cages to a point with a controlled total length. Each of these ways of contracting 1-cages leads to a 1-parameter family of cages, that begins with the original cage and ends with a point. For every weighted length functional on the space of $i$-cages we assign the following weighted length decreasing process on the same space: For a given $i$-cage consider the weighted $i$-cage, where all edges acquire weights coming from the definition of the weighted length functional. Apply the "usual" length decreasing process (see Section 4) to this weighted $i$-cage obtaining a homotopy that ends at a (possibly trivial) critical point of the (usual) length functional. Then we drop the assigned weights, and obtain a homotopy in the space of $i$-cages that starts from the original $i$-cage. The value of the weighted length functional decreases along trajectories of this homotopy. The homotopy ends at a (possibly trivial) critical point of the weighted length functional.

Principle 3 For every 1-parameter family of $k$-cages described in 2 there is a $k$-disc that "fills" it. If not, we would either obtained infinitely many geometrically distinct geodesic nets or a short periodic geodesic on the $k$ th step of our inductive construction. This allows us to extend to the $k$-skeleton, $k \leq q$. This $k$-disk is obtained from $(k-1)$-spheres filling individual $k$-cages in the considered 1 -parametric family. These $(k-1)$-spheres are obtained by glueing $(k-1)$-discs fillings $(k-1)$-subcages of the $k$-cages.

Principle 4 Discs are constructed from spheres (of one dimension smaller), spheres are constructed from discs (of the same dimension). A $k$-disc is constructed by producing a 1-parameter family of $(k-1)$-spheres that start with the original sphere and end with a point. This family of spheres is created by contracting the original $k$-cage to a point (using an assumption that there is no "small" geodesic cages) and at each time constructing a $(k-1)$-sphere, as it was discussed before. A sphere is obtained by gluing discs, just as we glue $(k-1)$-dimensional simplices in the boundary of a $k$-simplex to obtain a sphere (however, since one of those simplices is small, we treat it as a point). These discs are obtained from $(k-1)$-cages. For example, when we extend to 3-skeleton (see Figure 5), three discs are obtained from 3-cages as one parameter family of 2-spheres. 2-spheres are obtained by gluing three 2-discs, that 
are constructed by applying the BCSP to the three digons obtained at each $\tau, \tau \in[0,1]$ from a 3 -cage as its 2 -subcages.

Principle 5 Continuity is important. So, after we have extended to $k$-skeleton, using, let's say, the cage shortening process associated with $L^{k}\left(m_{j}^{k}\right)$, every time we have to "move" a $k$-cage, we have to use the same cage shortening process. Thus, from each "live" $m_{j}^{3}$-branch there are growing infinitely many $m_{j}^{4}$-branches. Also from each

"live" $m_{j}^{4}$-branch there are growing infinitely many $m_{j}^{5}$-branches, etc. Here we also use a fact, that in the absence of minimal objects those spheres will change continuously. This proof uses a length shortening process for $k$-cages, which is an adaptation of a general length shortening process introduced in [9]. A simplified version is also used in $[10 ; 12 ; 13]$. For the sake of completeness we discuss how to adapt the process of [9] to the case of $k$-cages in Section 2.

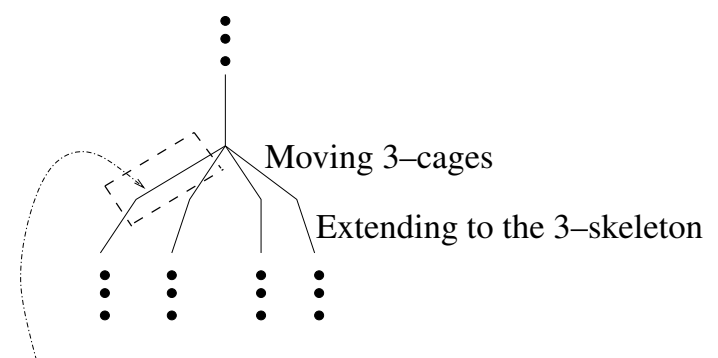

Let us look more closely at how moving 3-cages leads to extending to the 3 -skeleton.



Figure 5: The scheme of the proof

Theorem 0.3 will be proved in Section 2. In Section 3 we will prove Theorem 0.4. The proof is based on the combination of the ideas from the proof of Theorem 0.3 and the idea by M Gromov from [5] involving filling $M^{n}$ by a polyhedron $W^{n+1}$ in $L^{\infty}\left(M^{n}\right)$, attempting to extend the identity map on $M^{n}$ to $W^{n+1}$ and obtaining a desired extremal object (in our case a geodesic net) as an obstruction to this extension. 


\section{The proof of Theorem 0.3}

Lemma 2.1 (a) Let $M^{n}$ be a closed Riemannian manifold of dimension $n$. There exist infinitely many different weighted length functionals $L^{k}\left(m_{j}^{k}\right)$ on the space of $k$-cages, where $m_{j}^{k}$ are natural numbers, and $j=0,1,2, \ldots$, with the following properties:

(1) Consider two critical points of two distinct weighted length functionals from the considered sequence. Assume that the support of at least one of these critical points is not a periodic geodesic. Multiply the edges of these critical points by the weights of the corresponding weighted length functionals to obtain two critical points of the "usual" length functional. Then these two critical points of the length functional are geometrically distinct stationary geodesic cages.

(2) The weights of $L^{k}\left(m_{j}^{k}\right)$ are equal to $m_{j}^{k}+c_{1}, \ldots, m_{j}^{k}+c_{k}$ for appropriately chosen numbers $c_{1}, \ldots, c_{k}$. More specifically, one can take $c_{i}=k^{2 i}, i=1, \ldots, k$ and $\left\{m_{j}^{k}\right\}_{j=1}^{\infty}$ a cofinite subsequence of the sequence $\left\{\left(1+2 k^{4 k+3}\right)^{j}\right\}_{j=1}^{\infty}$.

(b) Let $L^{k}\left(m_{j}^{k}\right)$ denote the weighted length functionals introduced in part (a) of the lemma, and $d$ denotes the diameter of $M^{n}$. Assume that $L^{k}\left(m_{j}^{k}\right)$ has a critical point $c$, such that $L^{k}\left(m_{j}\right)(c) \leq\left(\sum_{i=i}^{k} c_{i}+k m_{j}^{k}\right) d$. Then the length of the support of $c$ does not exceed $k d+\frac{\Sigma_{i=1}^{k} c_{i}}{m_{j}^{k}} d=\left(k+O\left(\frac{1}{m_{j}^{k}}\right)\right) d=k d+o(1)$, as $j \longrightarrow \infty$.

Proof (a) Consider the weighted length functional $L^{k}\left(m_{j}^{k}\right)$ with weights $\left(m_{j}^{k}+\right.$ $\left.c_{1}, \ldots, m_{j}^{k}+c_{k}\right)$, where $c_{i}$ is a natural number. We would like to find a sequence $\left\{m_{j}^{k}\right\}_{j=0}^{\infty}$ and numbers $c_{i}$, such that the corresponding functionals have different critical points unless they are closed geodesics.

Let us note that during the length shortening process one or both of the following two things can happen: (1) two or more edges can become one; (2) one or more edges can shrink to a point. In the later case, the cage will degenerate into a flower.

Now let us consider two functionals $L^{k}(\eta)$ and $L^{k}(m)$, where $m=m_{j_{1}}^{k}$ and $\eta=m_{j_{2}}^{k}$ for some distinct $j_{1}$ and $j_{2}$. We can also assume that $m<\eta$. Suppose that two critical points of the length functional that are not geometrically distinct arise from critical points of $L^{k}(\eta)$ and $L^{k}(m)$. Let us denote the edges forming the carriers of these critical points of the length functional by $e_{1}, \ldots, e_{s}$, where $s \leq k$.

Let $a_{i} \eta+d_{i}$ denote the multiplicity of $e_{i}$ when we regard it as an edge in the critical cage corresponding to $L^{k}(\eta)$, and $b_{i} m+f_{i}$ the multiplicity of $e_{i}$ when regarded as an 
edge in the critical cage corresponding to $L^{k}(m), i=1, \ldots, s$. These notations imply that $a_{i}$ and $b_{i}$ are less than or equal to $k, d_{i}$ is the sum of some $a_{i}$ distinct numbers from the set $\left\{c_{1}, \ldots, c_{k}\right\}$ and $f_{i}$ is the sum of some $b_{i}$ distinct numbers from the same set. Note that if the crtitical net is not a closed geodesic, then $s \geq 2$. The condition that the critical points of the length functional corresponding to $L^{k}(m)$ and $L^{k}(\eta)$ are not geometrically distinct means that the multiplicities satisfy the following $s$ equations:

$$
a_{i} \eta+d_{i}=\alpha\left(b_{i} m+f_{i}\right), i=1, \ldots, s
$$

where $\alpha$ is a positive rational proportionality constant. Let us take a look at the first two such equations. The first one implies that $\eta=\frac{\alpha\left(b_{1} m+f_{1}\right)-d_{1}}{a_{1}}$. Combined with the second equation we will have $\alpha\left(a_{2}\left(b_{1} m+f_{1}\right)-a_{1}\left(b_{2} m+f_{2}\right)\right)=a_{2} d_{1}-a_{1} d_{2}$, where $a_{i}, b_{i}, f_{i}, d_{i}, m, \eta$ are all integers. Unless $a_{2}\left(b_{1} m+f_{1}\right)-a_{1}\left(b_{2} m+f_{2}\right)=0$, $\alpha=\frac{a_{2} d_{1}-a_{1} d_{2}}{a_{2}\left(b_{1} m+f_{1}\right)-a_{1}\left(b_{2} m+f_{2}\right)} \leq \max \left\{a_{2} d_{1}, a_{1} d_{2}\right\} \leq k^{2} \max _{i=1}^{k} c_{i}$. Therefore, $\eta \leq$ $k^{2} \max _{i=1}^{k} c_{i}\left(k m+k \max _{i=1}^{k} c_{i}\right) \leq 2 k^{3}\left(\max _{i=1}^{k} c_{i}\right)^{2} m$.

To ensure that this case is impossible we can define the sequence $\left\{m_{j}^{k}\right\}_{j=1}^{\infty}$ as an arbitrary cofinite subsequence of the sequence $\left\{\left(1+2 k^{3}\left(\max _{i=1}^{k} c_{i}\right)^{2}\right)^{j}\right\}_{j=1}^{\infty}$.

Now let us examine the case when $a_{2}\left(b_{1} m+f_{1}\right)-a_{1}\left(b_{2} m+f_{2}\right)=0$. This equation has more than one solution $m\left(a_{1}, a_{2}, b_{1}, b_{2}, f_{1}, f_{2}\right)$ if and only if $f_{1}=\frac{a_{1} f_{2}}{a_{2}}$ and $b_{1}=\frac{a_{1} b_{2}}{a_{2}}$. We claim that we can easily find coefficients $c_{1}, . ., c_{k}$, so that it never happens. Recall that $a_{i}$ and $b_{i}$ are just number of different edges merging into the considered edge. The numbers $f_{1}$ and $f_{2}$ are sums of $c_{j}$ components of the weights of the merging edges. Therefore, let $c_{1}=k^{2}, \ldots, c_{i}=k^{2 i}, \ldots, c_{k}=k^{2 k}$. Without loss of generality, assume that $b_{2}>b_{1}$. Then $f_{2}>f_{1}$. Note that $\frac{b_{2}}{b_{1}} \leq k$. On the other hand, let us examine $\frac{f_{2}}{f_{1}}$. For some $r \leq k \frac{f_{2}}{f_{1}}>\frac{k^{2 r}}{(k-1) k^{2(r-1)}}>k$. Therefore, in such a case it cannot happen that $\frac{f_{2}}{f_{1}}=\frac{b_{2}}{b_{1}}$. Thus, for chosen $c_{1}, \ldots, c_{k}$, the total number of all solutions $m$ of $a_{2}\left(b_{1} m+f_{1}\right)-a_{1}\left(b_{2} m+f_{2}\right)=0$ does not exceed the number of all possible six tuples $\left(a_{1}, a_{2}, b_{1}, b_{2}, f_{1}, f_{2}\right)$, which does not exceed $k^{2} 2^{4 k}$. Therefore, for every $k$ and for all, but finitely many values of $j m_{j}^{k}=\left(1+2 k^{4 k+3}\right)^{j}$ correspond to geometrically distinct critical nets. So, there exists a cofinite subsequence $m_{j}^{k}$ of the sequence $\left\{\left(1+2 k^{4 k+3}\right)^{j}\right\}_{j=1}^{\infty}$ satsfying the conditions of Lemma 2.1 (a).

(b) Indeed, if a value of $L^{k}\left(m_{j}^{k}\right)$ at one of its critical points is equal to $x$, then the length of the underlying (unweighted) $k$-cage does not exceed $\frac{x}{m_{j}^{k}+c_{1}}$, whence the assertion of (b) immediately follows.

Proof of Theorem 0.3 The theorem will be proved by contradiction. Let $M^{n}$ be a closed Riemannian manifold, such that $\pi_{1}\left(M^{n}\right)=\ldots=\pi_{q-1}\left(M^{n}\right)=\{0\}$ and 
$\pi_{q}\left(M^{n}\right) \neq\{0\}$. Let $f: S^{q} \longrightarrow M^{n}$ be a non-contractible map of a finely triangulated sphere to $M^{n}$. Assuming there are no "small" periodic geodesics and only finetely many stationary geodesic nets, we will extend this map to the disc $D^{q+1}$ of dimension $q+1$, thus reaching a contradiction. To construct this extension, we will triangulate the disc as the cone over the chosen triangulation of the sphere. The procedure will then be inductive on skeleta of $D^{q+1}$. To begin with, the center of the disc will be mapped to an arbitrary point in $M^{n}$ and the edges will be mapped to minimal geodesic segments that connect this point with corresponding vertices of the triangulation of the image sphere. Finally, to extend to the 2-skeleton, we will consider simplices of the form $\widetilde{\sigma}_{i}^{2}$. The boundary is mapped to a closed curve of length $\leq 2 d+\delta$. As there are no short periodic geodesics, it can be contracted to a point using the regular Birkhoff curve shortening process. We will use the disc generated by this homotopy to extend to $\widetilde{\sigma}_{i}^{2}$.

The rest of the extension procedure uses two ideas:

(1) "Filling" $k$-cages by $k$-discs for all values of $k \leq q+1$, which is an inductive bootstrap procedure similar to the one used in [12; 13]: Assuming that we have extended our map to the $k$-skeleton, we will explain how to extend it to the $(k+1)$-skeleton of $D^{q+1}$. In order to do that we will extend $f$ to each $(k+1)$-dimensional simplex of $D^{q+1}$, and in order to do that it will be necessary to "fill" $(k+1)$-cages by $(k+1)-$ dimensional discs. Note that from the previous step of the induction we already know how to "fill" $(k+1)$-cages by $k$-spheres.

To explain the last assertion consider the image of the boundary of the above simplex. It consists of $k+2 k$-dimensional simplices, one of which is so small that it can be treated as a point (see Remark 4). Here the idea is that we can contract this simplex to a point over itself, reducing our situation to the situation, where the simplex is treated as a point. The remaining $k+1 k$-simplices were already obtained during the previous step of the extension process. Thus, the inductive step of the extension reduces to contracting $k$-spheres "filling" $k$-cages.

(2) One basic idea for contracting these $k$-spheres is that in the absence of infinitely many stationary geodesic nets and short periodic geodesics, there is a homotopy connecting the cage with a point. Because of the continuity of the filling as a function of the contracted cage, the $k$-sphere filling the cage also contracts to a point. Here are the details of our construction.

Suppose that we were able to extend the map $f: S^{q} \longrightarrow M^{n}$ to the $k$-skeleton of $D^{q+1}$ proceeding as in the previous step of the induction process. In fact, there are infinitely many such extensions, corresponding to infinitely many weighted length functionals that were used for this purpose. Because of our construction and assumptions, an 
application of the weighted length shortening process to any $k$-cage with support of total length $\leq k d$ (in fact $\leq(q+1) d$ ) contracts this $k$-cage to a point for any of these functionals . Moreover, the contracting homotopy will depend continuously on the $k$-cage that is being contracted. Now we can just fix one of such weighted length functionals, $L^{k}$. We can assume that similar weighted length functionals $L^{i}$ on spaces of $i$-cages were already fixed for all $i<k$.

Our choice of the finite sequence of weighted length functionals $L^{i}$ corresponds to one particular extension of $f$ to the $k$-skeleton of the chosen triangulation of $D^{q+1}$. We will extend this extension to the $(k+1)$-skeleton. (This can be done also in infinitely many distinct ways.) That will be done as follows: let $\widetilde{\sigma}_{i}^{k+1}$ be a $k+1-$ simplex in the triangulation of $D^{q+1}$. Consider a $(k+1)$-cage $C g$ that corresponds to the image of 1 -skeleton of this simplex. Apply a weighted length shortening process $L^{k+1}\left(m_{j}^{k+1}\right)$ for $m_{j}^{k+1}$ and $c_{i}$ constructed as in Lemma 2.1. Recall that we assumed that there are no non-trivial periodic closed geodesics of length $\leq(q+1) d$, and there are only finitely many geometrically distinct stationary $1-$ cycles. Lemma 2.1 implies that infinitely many of such length-shortening processes will lead to a homotopy that contracts every $(k+1)$-cage of length $\leq(k+1) d$ (and even of length $\leq(q+1) d)$ to a point. This homotopy will be a continuous function of the initial $(k+1)$-cage. We can choose one of the corresponding weighted length functionals $L^{k+1}\left(m_{j}^{k+1}\right)$ to be $L^{k+1}$.

Consider the weighted length shortening process determined by $L^{k+1}$. During this process a $(k+1)$-cage (of length $\leq(k+1) d)$ is contracted to a point along a 1parameter family of $(k+1)$-cages $C g_{\tau}, \tau \in[0,1]$ of smaller weighted length. We can now construct a 1 -parameter family of spheres $S_{\tau}^{k}$ of dimension $k$ that starts with the image of the boundary of the given simplex and ends with a point, and thus generates a $(k+1)$-dimensional disc. Spheres are constructed by the procedure of "filling" cages $C g_{\tau}$ at each $\tau$ described in [12]. That is, given a $(k+1)$-cage $C g_{\tau}$, consider its $k+1$ " $k$-subcages", ie, $k$-tuples $\left(e_{1}\right)_{\tau}, \ldots,\left(\hat{e}_{j}\right)_{\tau}, \ldots,\left(e_{k+1}\right)_{\tau}$ obtained by ignoring one of the curves. By induction assumption, each of these subcages can be "filled" by discs of dimension $k$. (The base of induction is proved by contracting 2-cages, ie, closed curves, by the usual Birkhoff curve shortening process. At this point we are using the assumption that there are no short periodic geodesics. More precisely, Lemma 2.1 implies that we need to contract digons of length $\leq(k+1) d+O\left(\frac{1}{m_{k+1}^{j}}\right)$. If we get stuck at a closed geodesic for an infinite sequence of values of $j$, we can choose a subsequence of the sequence of these closed geodesics converging to a closed geodesic of length $\leq(k+1) d \leq(q+1) d$ obtaining a contradiction.) The weights that we are using in order to fill $k$-cages (and, more generally, $(k-i)$-cages) are already fixed on the previous steps of the filling procedure. These choices are coded by a path 
from the root (depicted at the top) of the tree on Figure 4 to the vertex of this tree that we are considering at the moment. Because of our previous choices of the weighted length functionals $L^{3}, L^{4}$, etc. we can be sure that we are not going to get stuck at non-trivial critical points of these functionals.

We then glue the resulting $(k+1)(k+1)$-discs as in the boundary of a $(k+1)$-simplex to obtain $S_{\tau}^{k}$. (We take the $(k+2)$ nd $k$-disc in the boundary of a $(k+1)$-simplex to be trivial.)

It is important to note that the just described process of "filling" of $(k+1)$-cages $C g_{\tau}$ by $k$-spheres is continuous with respect to $C g_{\tau}$ (and therefore with respect to $\tau$ ). The resulting one-parameter family of $k$-spheres generates the desired $(k+1)$-dimensional disc that can be used to extend $f$. (Indeed, since $C g_{1}$ is the trivial $(k+1)-$ cage, the corresponding $k$-sphere is also trivial.)

Remark 4 Let us consider a sphere in the manifold $M^{n}$ obtained as follows: Take a small 2-simplex $\left[v_{i_{1}}, v_{i_{2}}, v_{i_{3}}\right]$ and a point $p$, connect $p$ with each $v_{i_{j}}$ by a minimal geodesic segment $e_{j}, j=1,2,3$, and finally, contract each of the closed curves $e_{j}+$ $\left[v_{i_{j}}, v_{i_{j \bmod 3+1}}\right]-e_{j \bmod 3+1}$, where $j=1,2,3$ to a point by a length non-increasing homotopy (see Figure 6 (a)). (Of course, here we use the assumption that there are no closed geodesics of length $\leq 2 d+\varepsilon$, where $\varepsilon$ can be as large as $d$.) In the proof of Theorem 0.3 we claimed that the simplex $\left[v_{i_{1}}, v_{i_{2}}, v_{i_{3}}\right]$ can be treated a point $q$. Here is a formal justification of this claim:

Choose a point $q \in\left[v_{i_{1}}, v_{i_{2}}, v_{i_{3}}\right]$. Consider the boundary $\partial\left[v_{i_{1}}, v_{i_{2}}, v_{i_{3}}\right]=\left[v_{i_{2}}, v_{i_{3}}\right]-$ $\left[v_{i_{1}}, v_{i_{3}}\right]+\left[v_{i_{1}}, v_{i_{2}}\right]$. Denote each of the segments $\left[v_{i_{j}}, v_{i_{j \bmod 3+1}}\right]$ as $s_{j}, j=1,2,3$. Without any loss of generality, we can assume that $s_{1}+s_{2}+s_{3}$ can be contracted to $q$ in $\left[v_{i_{1}}, v_{i_{2}}, v_{i_{3}}\right]$ without the length increase. In addition, we can assume that the length of a trajectory, $\sigma_{j}$, of $v_{i_{j}}$ during this homotopy does not exceed a small number $\varepsilon(\delta)$, where $\varepsilon(\delta) \longrightarrow 0$ as $\delta \longrightarrow 0$. Denote the images of $s_{j}$ under this homotopy at the time $t$ as $s_{j_{t}}$, and the trajectories of $v_{i_{j}}$ traced when the time varies from 0 to $t,(t \leq 1)$, as $\sigma_{j_{t}}$ For every $t \in[0,1]$ we now can consider curves $e_{j}+\sigma_{j_{t}}+s_{j_{t}}-\sigma_{j \bmod 3+1_{t}}-e_{j} \bmod 3+1$ of length $\leq 2 d+2 \varepsilon(\delta)+3 \delta$ (see Figure 6 (b), (c)). Each of those curves is contractible to a point without the length increase, assuming there are no geodesics of length $\leq 3 d$. Moreover, at $t=1$ we will obtain a sphere $\Sigma$ that is constructed as follows: take two points $p$ and $q$ and connect them with three segments $e_{j}^{*}=e_{j}+\sigma_{j}, j=1,2,3$. Then take three digons $e_{j}^{*}-e_{j \bmod 3+1}^{*}$ and contract them to the point by the length decreasing homotopy, (see Figure $6(\mathrm{~d})$ ). We already demonstrated that the initial 2 -sphere and the final 2-sphere, $\Sigma$, are homotopic. Therefore, if $\Sigma$ is contractible, then our initial 2-sphere is contractible as well. The same argument straightforwardly generalizes for higher dimensions. 


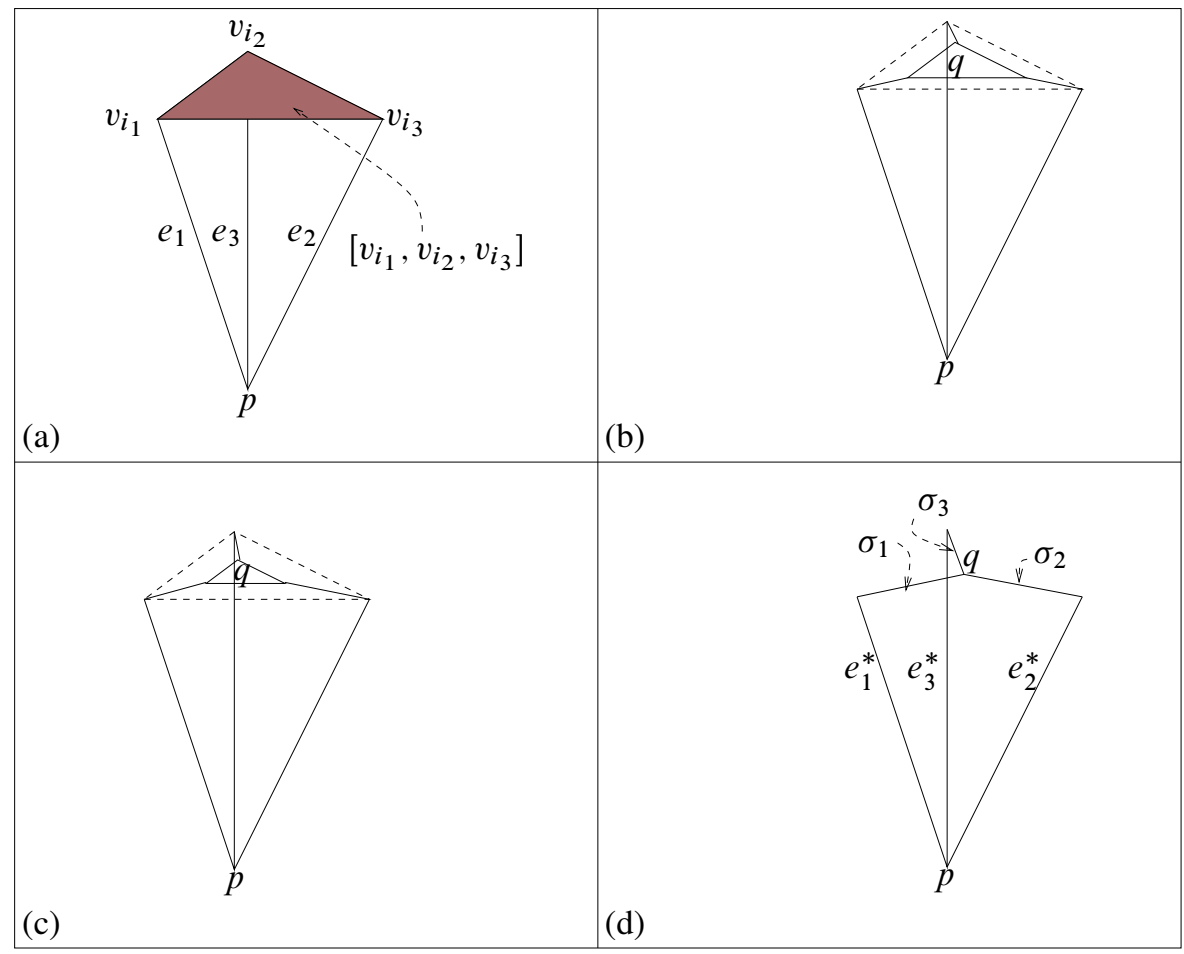

Figure 6: A small 2-simplex can be ignored

Remark 5 Note that if $l>(k+1) d$, then we can choose explicit values $m_{j}^{k}$ sufficiently large to ensure that our curve and cage shortening homotopies never get stuck at nontrivial periodic geodesics. This observation easily implies Remark 1 after Theorem 0.3 .

Remark 6 As we mentioned in the introduction, in the absence of short periodic geodesic there exists infinitely many stationary geodesic flowers. More precisely:

Theorem 2.2 Let $M^{n}$ be a closed Riemannian manifold of diameter $d$. Let $q=$ $\min _{i}\left\{\pi_{i}\left(M^{n}\right) \neq 0\right\}$. Then either there exists a non-trivial periodic geodesic on $M^{n}$ of length $\leq 2 q d$, or there exist infinitely many stationary geodesic flowers with carriers consisting of at most $q$ geodesic loops emanating from the same point. In the second case for every positive $\delta$ one can choose these stationary geodesic flowers so that the length of their support is $\leq 2 q d+\delta$.

Corollary 2.3 Assume that $M^{n}$ is a simply-connected closed Riemannian manifold of diameter $d$ with a non-trivial second homology group. Then either there exists a 
non-trivial periodic geodesic on $M^{n}$ of length $\leq 4 d$, or for every positive $\delta$ there exist infinitely many stationary geodesic flowers with carriers consisting of two geodesic loops emanating from the same point. In the second case for every positive $\delta$ one can choose these stationary geodesic flowers so that the length of their support is $\leq 4 d+\delta$.

Idea of the proof of Theorem 2.2 We combine our proof of Theorem 0.3 with the main idea of [13]. Instead of defining weighted length functionals $L^{k}\left(m_{j}^{k}\right)$ as in Lemma 2.1 we define them as follows: The weights of the first $(k-1)$ edges as the same as in Lemma 2.1, and the weight of the last edge is equal to the sum of the first $(k-1)$ weights. It is easy to see that for $k>2$ Lemma 2.1 will remain true for these functionals. Then we can proceed exactly as in the proof of Theorem 0.3. Our choice of weights implies that every stationary point of these functionals must be a $(k-1)$-flower, since the $k$ th edge must collapse to a point. (Otherwise, there is no way to balance the weighted sum of the unit tangent vectors to the edges of the cage at its vertices.) The constant $2 q$ (in $2 q d$ ) appears as the limit of the ratio of the sum of the weights of $L^{q+1}\left(m_{j}^{q+1}\right)$ to the smallest of these weights, as $j \longrightarrow \infty$.

Remark 7 Note that our definition of geometrically distinct stationary cages is weaker than the requirement that their supports are distinct sets. (Two stationary geodesic nets could have identical supports and even identical carriers but non-collinear multiplicity vectors.) Yet an easy argument shows that for almost all analytic Riemannian metrics on an underlying manifold our definition of geometrically distinct stationary cages is equivalent to the condition that their supports are distinct providing that two stationary geodesic cages that are being compared are not periodic geodesics. (As usual, "almost all" means that the considered set of metrics is the complement to a countable union of nowhere dense sets.) So, for almost all analytic Riemannian metrics we obtain either a short periodic geodesic or infinitely many geodesic cages as in Theorem 0.3 (or in Theorem 0.4) which have distinct supports. In the remaining (exceptional) cases we obtain either a short periodic geodesic or an infinite sequence of stationary geodesic cages as in Theorem 0.3 (or Theorem 2.2) with identical carriers but pairwise noncollinear multiplicity vectors. The last situation obviously cannot occur if the carrier consists of just two geodesic loops emanating from the same point. Therefore in the situation of Corollary 2.3 we always obtain either a periodic geodesic of length $\leq 4 d$ or infinitely many stationary geodesic flowers supported at distinct 2 -flowers of length $\leq 4 d+\delta$.

The proof of Corollary 2.3 easily implies that an infinite sequence of these 2-flowers will converge to a stationary 2 -flower of length $\leq 4 d$. (The existence of a stationary 2 -flower of length $\leq 4 d$ in the considered situation is already known [9]. Similarly, the existence of stationary cages and flowers satisfying the same length bounds as the 
stationary cages and flowers that could be obtained by a similar limit construction in the general situation had been proven in $[12 ; 13]$. Yet this observation suggests the following question: Is it possible to obtain infinitely many stationary flowers supported at "short" 2-flowers by perturbing one "short" stationary geodesic 2-flower? (And in the case of success one could try to reprove our main results in the general case similarly perturbing a non-trivial stationary cage or flower obtained in $[12 ; 13]$. Such a proof of our main results would be conceptually simpler, and would produce infinitely many stationary geodesic cages that are not merely geometrically distinct but have distinct supports.) Yet this idea encounters significant (and maybe even unsurmountable) difficulties. The first of them is that the vertex of a stationary 2 -flower can be conjugate to itself along one of the geodesic loops forming the 2-flower. As the result, small perturbation of the vertex of the 2-flower do not always extend to perturbations of both geodesic loops.

\section{The proof of Theorem 0.4}

Theorem 0.4 is proved by similar methods. However, we will need the definition of the Filling Radius below originally defined by M Gromov in [5].

Definition 3.1 [5] Let $M^{n}$ be an abstract manifold and let $X=L^{\infty}\left(M^{n}\right)$ be the Banach space of bounded Borel functions $f$ on $M^{n}$. Let $M^{n}$ be isometrically imbedded in $X$, where the imbedding of $M^{n}$ into $X$ is the map that assigns to each point $p$ of $M^{n}$ the distance function $p \longrightarrow f_{p}=d(p, q)$. Then the filling radius FillRad $M^{n}$ is the infimum of $\varepsilon>0$, such that $M^{n}$ bounds in the $\varepsilon$-neighborhood $N_{\varepsilon}\left(M^{n}\right)$, ie, homomorphism $H_{n}\left(M^{n}\right) \longrightarrow H_{n}\left(N_{\varepsilon}\left(M^{n}\right)\right)$ vanishes, where $H_{n}\left(M^{n}\right)$ denotes the singular homology group of dimension $n$ with coefficients in $\mathbb{Z}$, when $M$ is orientable, and with coefficients in $\mathbb{Z}_{2}$, when $M$ is not orientable.

Alternatively, one can give a different definition of the filling radius of $M^{n}$ by defining first FillRad $\left(M^{n} \subset X\right)$, the filling radius of $M^{n}$ isometrically imbedded into some metric space $X$, as the smallest $\varepsilon$, such that $M^{n}$ bounds in the $\varepsilon$-neighborhood of $M^{n}$ and then taking the infimum over all of the isometric imbeddings. It was shown by M Katz that FillRad $M^{n} \leq \frac{d}{3}$, where $d$ is the diameter of $M^{n}$ (see [8]).

In [5] M Gromov had found an estimate for the filling radius of a closed Riemannian manifold in terms of the volume of this manifold.

Theorem 3.2 [5] Let $M^{n}$ be a closed connected Riemannian manifold. Then FillRad $M^{n} \leq g c(n)\left(\operatorname{vol}\left(M^{n}\right)\right)^{\frac{1}{n}}$, where $g c(n)=(n+1) n^{n}(n+1) !^{\frac{1}{2}}$ and $\operatorname{vol}\left(M^{n}\right)$ denotes the volume of $M^{n}$. 
In this section we will prove the following:

Theorem 3.3 Let $M^{n}$ be a closed Riemannian manifold. Then either there exists a periodic geodesic on $M^{n}$ of length $\leq a(n)$ FillRad $M^{n}$ or there exist infinitely many geometrically distinct geodesic nets. Assume that the length $l$ of the shortest periodic geodesic is greater than $a(n) \operatorname{FillRad}\left(M^{n}\right)$. There exists a function $A(n, k), k \in\{1,2,3, \ldots\}$, such that for every $k$ there exist $k$ geometrically distinct stationary geodesic nets of length $\leq A(n, k)$ FillRad $M^{n}$. Moreover, one can write explicit formulae for $a(n)$ and $A(n, k)$.

Theorem 3.3 combined with Theorem 3.2 leads to the volume bound in Theorem 0.4.

Proof of Theorem 0.4 The proof of Theorem 3.3 is based on the combination of the ideas from the proof of Theorem 0.3 and an adaptation of the trick by M Gromov from [5] involving filling $M^{n}$ by a polyhedron $W^{n+1}$ in $L^{\infty}\left(M^{n}\right)$, attempting to extend the identity map on $M^{n}$ to $W^{n+1}$ and otaining a short periodic geodesic or infinitely many geometrically distinct stationary geodesic nets as an obstruction to this extension.

The details of the proof of Theorem 3.3 are very similar to that of Theorem 0.3 , except that instead of contracting $k$-cages, we will be contracting 1 -skeletons of simplices. The spheres and discs are then built out of those 1-skeletons in a similar fashion. Also, for each $k$ the weighted length functionals applied to 1-skeletal net will be $L^{\sigma^{k}}\left(m_{j}^{k}\right)$ with weights $m_{j}^{k}+c_{1}^{k}, \ldots, m_{j}^{k}+c_{\frac{k(k+1)}{2}}^{k}$, where the weight $m_{j}^{k}+c_{i}^{k}$ corresponds to an edge $e_{i}$ of the 1 -skeleton of the $k$-dimensional simplex $\sigma^{k}$. One can then prove a lemma similar to Lemma 2.1 that one can find constants $c_{i}^{k}$ and a sequence $m_{j}^{k}$, such that each such length functional has geometrically distinct critical points, unless it is a periodic geodesic.

Here are some of the details of the extension process. Suppose the conclusion of Theorem 0.4 is not satisfied, that is there is only finitely many geometrically distinct minimal geodesic nets and no "short" periodic geodesics.

Definition 3.1 implies $M^{n}$ bounds a polyhedron $W$ in the (FillRad $\left.M^{n}+\delta\right)$-neighborhood of $M^{n}$ (see [5]). That is, $M^{n}=\partial W$, when $M^{n}$ is orientable and $M^{n}=\partial W$ mod 2, when $M^{n}$ is not orientable. Triangulate $W$ and $M^{n}$ so that the diameter of every simplex in this triangulation is smaller than some small $\delta>0$ and so that the triangulation of $M^{n}$ extends the triangulation of $W$. We will extend the identity map id: $M^{n} \longrightarrow M^{n}$ to $W$, thus reaching a contradiction.

The extension procedure will be inductive to skeleta of $W$. It is different from the extension procedure employed in the proof of Theorem 0.3 only in the initial stages of extending to the 0,1 -skeleta of $W$. 


\section{The 0,1-skeleta}

Let us begin with the 0 -skeleton of $W$. To each vertex $\widetilde{w}_{i} \in W$ we will assign a vertex $w_{i} \in M^{n}$, that is closest to $\widetilde{w}_{i}$. As the result $d\left(\widetilde{w}_{i}, w_{i}\right) \leq$ FillRad $M^{n}+\delta$. Next, to extend to the 1-skeleton, we will assign to each edge of the form $\left[\widetilde{w}_{i}, \widetilde{w}_{j}\right] \subset W \backslash M^{n}$ a minimal geodesic segment $\left[w_{i}, w_{j}\right]$ connecting $w_{i}$ and $w_{j}$ of length $\leq 2 \operatorname{FillRad} M^{n}+$ $3 \delta$.

\section{The 2-skeleton}

Now, let us extend to the 2 -skeleton. Let $\widetilde{\sigma}_{i_{0}, i_{1}, i_{2}}^{2}=\left[\widetilde{w}_{i_{0}}, \widetilde{w}_{i_{1}}, \widetilde{w}_{i_{2}}\right]$ be an arbitrary $2-$ simplex. Its boundary is mapped to a closed curve of length $\leq 6$ FillRad $M^{n}+9 \delta$. This curve can be contracted to a point without the length increase, using the Birkhoff curve shortening process, assuming, of course, that there are no periodic geodesics of smaller length. Moreover, assuming that there are no "short" periodic geodesics, this homotopy depends continuously on the original curve. We will map $\widetilde{\sigma}_{i_{0}, i_{1}, i_{2}}^{2}$ using the above mentioned homotopy, obtaining a singular 2 -simplex that we will denote as $\sigma_{i_{0}, i_{1}, i_{2}}^{2}$.

\section{The 3-skeleton}

Next let us go to the 3-skeleton. Consider an arbitrary 3-simplex $\tilde{\sigma}_{i_{0}, i_{1}, i_{2}, i_{3}}^{3}=$ $\left[\widetilde{w}_{i_{0}}, \ldots, \widetilde{w}_{i_{3}}\right]$. By the previous step of the induction, its boundary is mapped as the following singular chain: $\Sigma_{j=0}^{3}(-1)^{j} \sigma_{i_{i}, \ldots, \hat{i}_{j}, \ldots, i_{3}}^{2}$. Consider its $1-$ skeleton. It will be a (not geodesic) net, that we will denote by $K_{i}$. Let us apply a weighted length shortening process $L^{\sigma^{3}}\left(m_{j}^{3}\right)$ to continuously deform it to a point. In fact, there exist infinitely many such weighted length functionals, as there are no "short" periodic geodesics and only finitely many distinct stationary nets. (The weighted length decreasing process for nets is defined similarly to its definition for cages: We multiply all edges of a net by weights coming from a considered weighted length functional and apply the "usual" length decreasing process described in the next section. Then we drop the weights and restore the original numeration of the edges.) We will be using weighted length functional $L^{k}\left(m_{j}^{k}\right)$ with weights $m_{j}^{k}+c_{i}, i \in\left\{1, \ldots, \frac{k(k+1)}{2}\right\}$, where $m_{j}^{k}$ and $c_{i}$ can be defined similarly to how it had been done for cages in the proof of Lemma 2.1.

At each time $\tau, \tau \in[0,1]$ during this deformation, we can use the net $\left(K_{i}\right)_{t}$ to construct a 2-dimensional sphere $S_{\tau}^{2}$ in a way that is analogous to the similar construction in the proof of Theorem 0.3. This 1-parameter family of 2-spheres can be regarded as a 3 -disc that we will denote as $\sigma_{i_{0}}^{3}, \ldots, i_{3}$. We will assign it to $\widetilde{\sigma}_{i_{0}}^{3}, \ldots, i_{3}$. We can continue in a similar fashion until we reach the $(n+1)$-skeleton of $W$, thus constructing a singular chain on $M^{n}$, that has the fundamental class $\left[M^{n}\right]$ as its boundary, and therefore, arriving at a contradiction. 


\section{Length shortening process for $m$-cages}

Recall that our definition of weighted length shortening processes for weighted length functionals on spaces of cages (or more generally nets) used a length shortening process for cages (correspondingly, nets) described in [9] (as well as in [12; 13] in the case of cages). We refer the reader to [9] for the details. The length shortening process described there is defined for 1-cycles. However, it works without any changes in the case of nets. For the sake of completeness here we will sketch this process for cages. A similar length shortening process for closed curves was introduced by G Birkhoff and is described in detail in [2, Section 2]. Consider the length functional on the space $C_{L}^{m}$ of $m$-cages of length $\leq L$. One can construct a flow on $C_{L}^{m}$ that decreases the length functional, assuming there is no stationary $m$-cages of length $\leq L$. Note that points can also be regarded as $m$-cages. We claim that in such a case there exists a deformation retraction of $C_{L}^{m}$ to $M^{n}$, (that is, $m$-cages of zero length), such that the length functional decreases along the trajectory of the deformation. Consider an $m$-cage consisting of two vertices $a$ and $b$ and $m$ curves $\alpha_{i}, i=1, \ldots, m$ that join those vertices.

The length shortening process we will describe is very similar to the Birkhoff Curve Shortening Process.

We will begin by replacing the curves $\alpha_{i}$ 's by piecewise geodesics. To do this, subdivide each of the curves into $N$ "small" segments of equal length $\leq \operatorname{Inj} \operatorname{Rad}\left(M^{n}\right) / 4$, where $\operatorname{Inj} \operatorname{Rad}\left(M^{n}\right)$ denotes the injectivity radius of $M^{n}$ and $N=\left[\frac{4 L}{\operatorname{InjRad} M^{n}}\right]+1$. Then we replace each small segment by the minimal geodesic segment. Clearly, the original $m-$ cage and the new piecewise geodesic $m$-cage will be homotopic by a length-decreasing homotopy. Moreover, this homotopy will continuously depend on the initial cage. (This observation is analogous to the starting point of Birkhoff Curve Shortening Process; see [2]).

Thus, we find a deformation retraction of $C_{L}^{m}$ to a finite dimensional space that we will denote $F C_{L}^{m}$, such that the length of an arbitrary segment of the cage does not increase during this deformation.

$F C_{L}^{m}$ can be regarded as a subset of $\left(M^{n}\right)^{N}$ for a sufficiently large $N$.

Let $C g^{m} \in F C_{L}^{m}$. We can define a vector of steepest descent tangent to $F C_{L}^{m}$ at $C g^{m}$. It will be defined as follows: consider all the vertices (ie, non-smooth points) of the $m$-cage. There will be many vertices, where two geodesic segments come together and two points $a$ and $b$, where $m$ geodesic segments come together. If $a=b$, there will be one point where $\leq 2 m$ tangent vectors come together. 
At each vertex consider the sum of the diverging unit vectors tangent to geodesic segments meeting at this vertex (see Figure 7). This collection of vectors tangent to $M^{n}$ constitutes the vector of the steepest descent for $C g^{m}$. Note also, that it will also "work" for $m$-cages that are sufficiently close to $C g^{m}$. That is, for any $m$-cage, sufficiently close to $C g^{m}$, if we parallel transport our vector to that $m$-cage, we will obtain a vector such that the first variation of the length functional in the direction of this vector will be negative. We can now choose a net in $C_{L}^{m}$, a locally finite cover of $C_{L}^{m}$ by open neighborhoods of points of this net, and a subordinate partition of unity, and construct a vector field on $F C_{L}^{m}$ such that the first variation of the length functional in the direction of this field is negative and $F C_{L}^{m}$ deforms to $F C_{0}^{m}$ in a finite time.

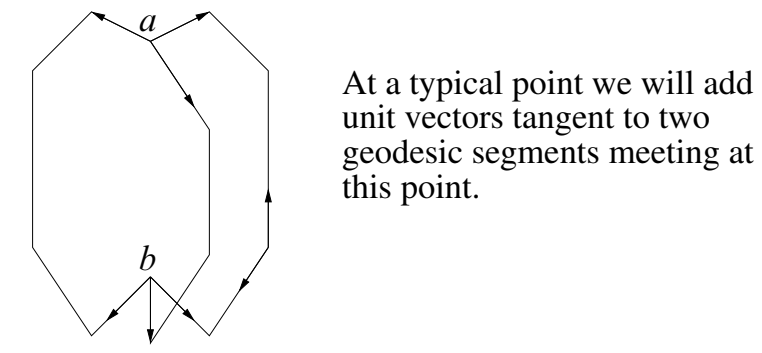

There will be three geodesic segments meeting at point $a$ and meeting at point $b$ so at each of these points we will have to add three unit vectors.

Figure 7: Length Shortening Process for $\theta$-graph

This process is a very much simplified version of the process described in paper [9] (see the proof of a Morse-theoretic type lemma for the space of 1-cycles made of at most $k$ segments [9, Lemma 3], in which we show that, assuming there are no non-trivial stationary 1 -cycles in the space of 1 -cycles $\Gamma_{k}^{x}$ made of at most $k$ segments of length $\leq x$, then the space $\Gamma_{k}^{0}$ of 1 -cycles of 0 length is a deformation retract of $\Gamma_{k}^{x}$ ). All the technical difficulties that arise during this deformation were dealt with in [9]. One can find an explanation of its simplified version that works for $\theta$-graphs in [10, Section $3]$. During this length shortening process, it can happen that the length of one of the edges becomes 0 and the two points $a$ and $b$ coincide. We will then have to move this unique vertex in the direction of the sum of all unit vectors tangent to geodesic segments and diverging from this vertex. This is the reason why the length shortening process needs to be defined using a locally finite covering of $F C_{m}^{L}$ and a subordinate partition of unity, rather than just the vector of the steepest descent. The vector of the 
steepest descent does not, in general, depend continuously on the cage near the cages, where $a=b$. Another difficulty is that despite the fact that the total length of each cage decreases, the distance between two neighboring vertices can increase. We want this distance to remain smaller than $\operatorname{InjRad} M^{n}$. Otherwise we will not be able to connect the endpoints by a unique geodesic segment. Therefore, to resolve this difficulty, we apply the flow only for the time $t=\frac{\operatorname{InjRad} M^{n}}{4}$. Then we stop, divide each segment into equal segments of length $\leq \frac{\operatorname{InjRad} M^{n}}{4}$ and replace it by a piecewise geodesic curve, as it was done in the beginning. Then we apply the flow again for $t=\frac{\operatorname{InjRad} M^{n}}{4}$ etc.

Under this curve shortening process the $m$-cage converges either to a stationary $m-$ cage (possibly degenerate, where two vertices coincide and lenghts of one or more segments are equal to zero) or to a point.

Acknowledgments This paper was partially written during the authors' visit to the Max-Planck Institute, Bonn. The authors would like to thank the Max-Planck Institute for its kind hospitality. Both authors gratefully acknowledge the partial support from Natural Sciences and Engineering Research Council (NSERC) Research Grants. Regina Rotman also acknowledges the partial support from NSERC University Faculty award. Both authors also gratefully acknowledge the partial support from their National Science Foundation research grants.

\section{References}

[1] V Bangert, On the existence of closed geodesics on two-spheres, Internat. J. Math. 4 (1993) 1-10 MR1209957

[2] C B Croke, Area and the length of the shortest closed geodesic, J. Differential Geom. 27 (1988) 1-21 MR918453

[3] J Franks, Geodesics on $S^{2}$ and periodic points of annulus homeomorphisms, Invent. Math. 108 (1992) 403-418 MR1161099

[4] D Gromoll, W Meyer, On differentiable functions with isolated critical points, Topology 8 (1969) 361-369 MR0246329

[5] M Gromov, Filling Riemannian manifolds, J. Differential Geom. 18 (1983) 1-147 MR697984

[6] J Hass, F Morgan, Geodesic nets on the 2-sphere, Proc. Amer. Math. Soc. 124 (1996) 3843-3850 MR1343696

[7] N Hingston, On the growth of the number of closed geodesics on the two-sphere, Internat. Math. Res. Notices (1993) 253-262 MR1240637 
[8] A B Katok, Ergodic perturbations of degenerate integrable Hamiltonian systems, Izv. Akad. Nauk SSSR Ser. Mat. 37 (1973) 539-576 MR0331425 English translation: Math. USSR Izv. 7 (1973) 535-571

[9] A Nabutovsky, R Rotman, Volume, diameter and the minimal mass of a stationary 1-cycle, Geom. Funct. Anal. 14 (2004) 748-790 MR2084979

[10] A Nabutovsky, R Rotman, The minimal length of a closed geodesic net on a Riemannian manifold with a nontrivial second homology group, Geom. Dedicata 113 (2005) 243-254 MR2171308

[11] H-B Rademacher, On the average indices of closed geodesics, J. Differential Geom. 29 (1989) 65-83 MR978076

[12] R Rotman, The length of a shortest geodesic net on a closed Riemannian manifold, Topology 46 (2007) 343-356

[13] R Rotman, Flowers on Riemannian manifolds, preprint Available at http:// www.mpim-bonn.mpg.de/preprints/retrieve

[14] M Vigué-Poirrier, D Sullivan, The homology theory of the closed geodesic problem, J. Differential Geometry 11 (1976) 633-644 MR0455028

[15] W Ziller, Geometry of the Katok examples, Ergodic Theory Dynam. Systems 3 (1983) 135-157 MR743032

AN, RR: Department of Mathematics, University of Toronto

Toronto, Ontario M5S 2E4, Canada

Department of Mathematics, Penn State University

University Park PA 16802, USA

Department of Mathematics, Penn State University

University Park PA 16802, USA

alex@math.toronto.edu, rina@math.toronto.edu

Proposed: Tobias Colding

Seconded: Leonid Polterovich, Jean-Pierre Otal

Received: 17 February 2007

Revised: 20 April 2007 